\title{
Functional Analysis of Keto-Acid Reductoisomerase ILVC in the Entomopathogenic Fungus Metarhizium robertsii
}

\author{
Yulong Wang ${ }^{1,2}$, Shihong Liu ${ }^{1,2}$, Xuebing Yin ${ }^{1,2}$, Deshui Yu ${ }^{1,2}$, Xiangyun Xie ${ }^{1,2}$ and Bo Huang ${ }^{1,2, *}$ \\ 1 Anhui Provincial Key Laboratory of Microbial Pest Control, Anhui Agricultural University, \\ Hefei 230036, China; wyl2019@ahau.edu.cn (Y.W.); lsh1491416840@163.com (S.L.); yxb@stu.ahau.edu.cn (X.Y.); \\ DeshuiYu@yeah.net (D.Y.); yuriname@163.com (X.X.) \\ 2 Engineering Research Center of Fungal Biotechnology, Ministry of Education, Anhui Agricultural University, \\ Hefei 230036, China \\ * Correspondence: bhuang@ahau.edu.cn; Tel./Fax: +86-551-6578-6887
}

\section{check for}

updates

Citation: Wang, Y.; Liu, S.; Yin, X.; Yu, D.; Xie, X.; Huang, B. Functional Analysis of Keto-Acid Reductoisomerase ILVC in the Entomopathogenic Fungus Metarhizium robertsii. J. Fungi 2021, 7, 737. https://doi.org/10.3390/ jof7090737

\section{Academic Editors:}

Sławomir Milewski and Ivan M. Dubovskiy

Received: 4 July 2021

Accepted: 6 September 2021

Published: 8 September 2021

Publisher's Note: MDPI stays neutra with regard to jurisdictional claims in published maps and institutional affiliations.

Copyright: (c) 2021 by the authors. Licensee MDPI, Basel, Switzerland. This article is an open access article distributed under the terms and conditions of the Creative Commons Attribution (CC BY) license (https:// creativecommons.org/licenses/by/ $4.0 /)$
Abstract: Ketol-acid reductoisomerase (ILVC) is the second enzyme in the branched-chain amino acid (BCAA) biosynthesis, which regulates many physiological activities in a variety of organisms from bacteria to fungi and plants. In this work, function mechanisms of ILVC in Metarhizium robertsii Metchnikoff (Hypocreales: Clavicipitaceae) were explored with site-directed mutagenesis, reductase activity assays and transcriptomics analysis. The reductase activity assays showed that ILVC from phytopathogenic fungi exhibited significantly higher activities than those from entomopathogenic fungi but lower than those from yeast. Site-directed mutagenesis and enzymatic activities of MrILVC with different active-site mutants (Arg-113, Ser-118, Asp-152, Asp-260, and Glu-264) confirmed that active sites of MrILVC are conserved with plant and bacterial ILVCs. Deleting MrilvC causes the complete failures of vegetative growth and conidial germination, feeding with branched-chain amino acids (BCAAs) recovers the fungal growth but not conidial germination, while both characteristics are restored when supplemented with yeast extract. Compared to $\triangle M$ rilv $\mathrm{C}$ cultured in czapek agar (CZA), plenty of genes involved in the biosynthesis of antibiotics and amino acids were up- or downregulated in the wild type or $\triangle M$ rilv C feeding with either BCAAs or yeast extract. Further analysis showed some genes, such as catalase $A$, participate in mycelial growth and conidial germination was down-regulated in $\triangle M$ rilv $C$ from CZA, revealing that MrILVC might control the fungal development by gene regulation and BCAAs or yeast extract could play partial roles of MrILVC. This study will advance our understanding of ILVC function mechanisms in fungi.

Keywords: Metarhizium robertsii; ILVC; reductase activity; transcriptomics analysis; mycelial growth; conidial germination

\section{Introduction}

Valine, leucine, and isoleucine form the small group of branched chain amino acids (BCAAs), which are synthesized in bacteria, plants, and fungi, but not in animals [1,2]. The biosynthesis of these amino acids shows high similarity between organisms of the different kingdoms of life, of which valine and isoleucine synthesis are carried out by the same enzymes, and leucine is created from $\alpha$-ketoisovalerate (a transamination precursor of valine) [3]. However, striking differences on the capabilities to synthesize or degrade branched-chain amino acids exist, and BCAAs in different groups of organisms also have some unique functions and features, revealing the various function mechanisms of BCAAs among different organisms [4].

The conservation in fungi but absence in mammals of the BCAA biosynthetic pathway makes it as the target for herbicides, fungicides, and antimicrobial compounds $[5,6]$. Acetohydroxyacid synthase (AHAS), the first enzyme in this pathway, has been targeted and inhibited by several commercial herbicides, such as sulfonanilides, imidazolinones, and sulfonylureas [7,8]. Ketol-acid reductoisomerase (ILVC, KARI, or ILV5), downstream 
of AHAS in the BCAA biosynthetic pathway, catalyzes the conversion of 2-acetolactate and 2-aceto-2-hydroxybutyrate to 2,3-dihydroxyisoverate and 2,3-dihydroxy-3-methylvalerate, respectively. Although there are no commercial herbicides targeting ILVC, some compounds have been reported as potent competitive inhibitors of ILVC in vitro, such as $\mathrm{HOE}$ 704, IpOHA, and CPD [6]. To date, BCAA enzyme inhibitors have shown antimicrobial effects on some harmful pathogenic microorganisms, including Fusarium graminearum Petch (Hypocreales: Nectriaceae), Pseudomonas aeruginosa Migula (Pseudomonadales: Pseudomonadaceae) and Candida albicans Berkhout (Saccharomycetales: Saccharomycetaceae), but whether they have inhibitory effects on pest pathogenic fungi are still uncharted $[6,9,10]$.

ILVC is a bifunctional enzyme that catalyzes two quite different reactions, but occur at a common active site, acting both as an isomerase and as a reductase [11]. ILVCs have a $G x G x x(G / A) x x x(G / A)$ motif as a binding site for $\operatorname{NADP}(\mathrm{H})$, and $\mathrm{Mg}^{2+}$ is required for binding $\operatorname{NADP}(H)[9,12,13]$. Site-directed mutagenesis and activity assays showed that plant and bacterial ILVCs have evolved different mechanisms of induced fit to prepare the active site for catalysis $[9,12]$. Previous sequence comparison revealed that those active residues are highly conserved across plant, fungal, and bacterial ILVCs, but the gene or genes responsible for ILVC activity in fungi have not been investigated in any detail. In addition, functional analysis of the ILVC has been carried out in bacteria, plants, and fungi; however, transcriptome analyses for the blocking of BCAA biosynthesis remain unclear $[6,14]$.

In our previous study, we found that ILVC is associated with conidial germination and fungal pathogenicity in the insect-pathogenic fungus Metarhizium robertsii Metchnikoff (Hypocreales: Clavicipitaceae), formerly classified as Metarhizium anisopliae, and the ilvC-deleted mutant failed to germinate on CZA complemented with three BCAAs. Complementation of BCAAs cannot recover the conidial germination for the ilv C-deficient strain [14]. This study seeks to further characterize ilvC in M. robertsii by phenotypic and comparative analyses of its deletion mutants. Relative catalytic activities of ilvC mutants in five active-site residues were assayed and catalytic activities of ILVCs from different fungi were compared.

\section{Materials and Methods}

\subsection{Phylogenetic Analysis}

BLAST searches were carried out to identify different eukaryotic ortholog of the Saccharomyces cerevisiae (GenBank accession number AJV63457.1) and Arabidopsis thaliana (NP_001078309.1) ILVC protein. ILVC sequences were aligned using ClustalX, and then a neighbor-joining (NJ) tree was generated using MEGA 7.0 software. ILVC sequences from Streptococcus pneumoniae (WP_000218054.1), Slackia exigua (WP_040619008.1), Alicyclobacillus acidocaldarius (WP_008340213.1), Mycobacterium tuberculosis (MXI72316.1), Pseudomonas aeruginosa (WP_070331278.1), Escherichia coli (MQL15051.1), A. thaliana, Spinacia oleracea (1QMG_A), Oryza sativa (EEE64772.1), M. robertsii (XP_007819198.1), F. graminearum (XP_011319056.1), Aspergillus fumigatus (XP_754177.1), and S. cerevisiae were used for phylogenetic analysis.

\subsection{Fungal Strain and Maintenance}

The ilvC-deficient $(\triangle M r i l v C)$, and complemented strains (Comp) were constructed from the wild-type (WT) M. robertsii strain ARSEF 23 (ATCC no. MYA-3075) in our previous report [14]. All strains grown on SDAY (4\% glucose, $1 \%$ peptone, $1 \%$ yeast extract powder, and $1.5 \%$ agar, $w / v$ ) at $28{ }^{\circ} \mathrm{C}$ in the dark. Conidia were harvested in a $0.05 \%$ Tween 80 aqueous solution from different strains cultured with 20 days and filtered through sterile nonwoven fabric for mycelia removal. The conidial concentration was determined using a hemocytometer and diluted to $1 \times 10^{7}$ conidia $\mathrm{mL}^{-1}$. 


\subsection{Site-Directed Mutagenesis, Protein Expression and Purification}

Site-directed mutagenesis was performed with TaKaRa MutanBest kit (TaKaRa, Beijing, China). Briefly, the ilvC sequence was amplified by PCR from the cDNA template and PCR products were inserted into pOT2 plasmid. pOT2-Ilvc plasmid was amplified with designed mutant primers by PCR, and blunted using Blunting Kination Enzyme Mix, then ligated using ligation solution I in the kit. Plasmids were transformed into Escherichia coli Migula (Enterobacterales: Enterobacteriaceas) DH5 $\alpha$ (TransGen, Beijing, China) and verified by DNA sequencing. Five amino acid residues contacting both $\mathrm{NADP}(\mathrm{H})$ and $\mathrm{Mg}^{2+}$ are conserved among bacteria, fungi, and plants; thus, their 5 active-site residue mutageneses were performed as mentioned above, respectively [15].

The recombinant ILVC protein was expressed in E. coli [16]. Briefly, ilvC with a $6 \times$ Histag sequence at the $\mathrm{C}$-terminus was amplified by PCR from the cDNA template or pOT2Ilvc, and PCR products were inserted into pET-28b (+) vector (Novagen, Beijing, China), then transformed into E. coli strain BL21 (DE3)-competent cells (TransGen, Beijing, China). ILVC protein expression was induced by the addition of isopropyl $\beta$-D-thiogalactoside (IPTG) to a final concentration of $0.5 \mathrm{mM}$ and purified with Ni-NTA agarose (Qiagen, Chatsworth, CA, USA) using a nickel-ion affinity column (Qiagen). Protein purity was monitored by SDS-PAGE.

\subsection{Reductase Activity Assays}

The enzymatic activity assays for ILVC protein were performed $[15,17,18]$. Firstly, ILVC protein $(1 \mu \mathrm{g})$ was pre-incubated in the activity assay buffer $(50 \mathrm{mM}$ Tris- $\mathrm{HCl} \mathrm{pH}$ 7.0, $1 \mathrm{mmol}$ DTT, $200 \mathrm{mmol} \mathrm{NADPH}, 10 \mathrm{mmol} \mathrm{MgCl}_{2}$ ) at room temperature for $30 \mathrm{~min}$. Secondly, 2-Acetolactate was added to the reaction mixture. The enzymatic activity was determined by monitoring the rate of NADPH oxidation at $340 \mathrm{~nm}$. Six independent experiments were repeated. In this study, reductase activity of ILVC from S. cerevisiae, A. fumigatus, F. graminearum, Beauveria bassiana (Hypocreales: Cordycipitaceae) and $M$. robertsii were determined by protein expression and purification from E. coli.

\subsection{The Sensitivity to Herbicides}

Mycelial growth tests were performed on SDAY plates supplemented with the following AHAS inhibitors at the concentrations of $10 \mu \mathrm{g} \mathrm{mL} \mathrm{m}^{-1}$ : tribenuron-methyl, rimsulfuron, metsulfuron-methyl, halosulfuron-methyl, chlorimuronethyl, imazethapyr and imazapyr (Henan Tianfu Chemical Co., Ltd., Henan, China). The percentage of inhibition of mycelial radial growth was calculated using the formula: mycelial growth inhibition $=[(C-N) /$ $(C-5)] \times 100$, where $C$ is colony diameter of the control, and $N$ is that of the treatment with AHAS inhibitors [6]. Each experiment was carried out with six replicates.

\subsection{Quantitative RT-PCR Analysis of MrilvC}

100- $\mu \mathrm{L}$ aliquots of a conidial suspension collected from strains cultured on SDAY were spread on SDAY at $28^{\circ} \mathrm{C}$ in the dark; at $18 \mathrm{~h}, 48 \mathrm{~h}$, and $96 \mathrm{~h}$, cultured strains were collected. Conidial suspensions were put on cicada (Cryptotympana atrata Westwood (Hemiptera: Cicadidae)) hind wings [19]. For the samples of M. robertsii during the infection progress, conidia were used for cuticle infection by dipping the fifth-instar Galleria mellonella L. (Lepidoptera: Pyralidae) larvae into a conidial suspension $\left(1 \times 10^{7}\right.$ conidia $\left.\mathrm{mL}^{-1}\right)$ for $10 \mathrm{~s}$. Then, the infected larvae were maintained in a stable photoperiod with $16 \mathrm{~h}$ of light and $8 \mathrm{~h}$ of dark at $28^{\circ} \mathrm{C}$ without feeding. Infected G. mellonella were collected at $24 \mathrm{~h}, 48 \mathrm{~h}$, and $96 \mathrm{~h}$. Quantitative RT-PCR was performed [20]. In brief, TRIzol reagent (Invitrogen, Carlsbad, CA, USA) was used for RNA extraction, and complementary DNA were synthesized with a gDNA remover kit (Toyobo, Osaka, Japan) as the manufacturer's instructions. Glyceraldehyde 3-phosphate dehydrogenase (GAPDH) was used as the control. Gene amplification was achieved using the Bio-Rad CFX96 real-time PCR detection system (Bio-Rad, Hercules, CA, USA). Transcripts of MrilvC were normalized to the control gene, 
and the $2^{-\Delta \Delta \mathrm{Ct}}$ method was used to calculate the relative expression level (mean \pm standard deviation) of each gene. Three independent experiments were performed for each gene.

\subsection{Phenotypic Assays}

A 4-mm-diameter agar plug of each strain from the border of a 5-day-old colonies on SDAY plates was centrally attached onto CZA $\left(0.3 \% \mathrm{NaNO}_{3}, 0.1 \% \mathrm{~K}_{2} \mathrm{HPO}_{4}, 0.05 \%\right.$ $\mathrm{MgSO}_{4} \cdot 7 \mathrm{H}_{2} \mathrm{O}, 0.05 \% \mathrm{KCl}, 0.001 \% \mathrm{FeSO}_{4} \cdot 7 \mathrm{H}_{2} \mathrm{O}, 3 \%$ sucrose, and $1.5 \%$ agar, $\left.w / v\right), \mathrm{CZA}+\mathrm{AA}$ (CZA with $1 \mathrm{mM}$ Ile, Leu, and Val) or CZA + yeast (CZA with $1 \%$ yeast extract powder, $w / v)$ at $28{ }^{\circ} \mathrm{C}$ under a $12 \mathrm{~h}: 12 \mathrm{~h}$ light/dark cycle. The radial growth (colony diameter) of the vegetative mycelia was measured daily from the back sides of the plates with vernier caliper. For the assessment of different strain conidial yield capacity, $100-\mu \mathrm{L}$ aliquots of a conidial suspension were spread on CZA, CZA + AA, and CZA + yeast. From day 3 onwards, 5-mm diameter colony disks were excised at random from the plates daily and washed off into $2 \mathrm{~mL}$ of $0.05 \%$. The concentration of the conidial suspension was determined by a hemocytometer under a microscope.

\subsection{Transcriptomics Analysis}

A 4-mm-diameter agar plug of each strain from the border of a 5-day-old colony containing both mycelium and conidium on SDAY plates was centrally attached onto $\mathrm{CZA}, \mathrm{CZA}+\mathrm{AA}$, and CZA + yeast plates for $24 \mathrm{~h}$ when both mycelial growth and conidial germination happened. RNA samples, library construction, and sequencing were performed [21]. Total RNA was extracted and treated with recombinant DNase I (rDNase I; Sigma, St Louis, MO, USA) to remove any potential genomic DNA contamination. To maximize target coverage, equal amounts of total RNA from the three replicates of strains were pooled as a sample library for RNA-Seq library construction, and then a total of $5 \mu \mathrm{g}$ of total RNA were prepared for RNA-Seq.

Total RNA was purified with oligo(dT) beads and fragmented into small pieces, treated with RNase H for RNA removement after the complementary DNA synthesized. The cDNA library was constructed and sequenced using the Illumina Hi-Seq 4000 platform with a single-end (single reads of 50 bases) sequencing strategy at Beijing Genomics Institute (BGI, Shenzhen, China). Clean reads were obtained by removing the raw reads, then used to map the M. robertsii genome (https: / / www.ncbi.nlm.nih.gov / genome/?term=Metarhizium+ robertsii (accessed on 31 January 2020)). The gene expression level was normalized using the fragments per kilobase per million mapped reads (FPKM) method [22]. Rigorous algorithms were applied to identify differential expressed genes (DEGs) with a false discovery rate (FDR) $\leq 0.001$ and an absolute value of the $\log _{2}$ ratio $\geq 1$ as thresholds [23]. Gene ontology (GO) functional analysis (Blast2GO, https: / /www.blast2go.org/ (accessed on 13 March 2020)) and Kyoto Encyclopedia of Genes and Genome (KEGG) pathway enrichment analysis was performed to further understand the biological functions of the identified DEGs. In addition, a total of 20 genes involved in conidial germination or mycelial growth in this study were selected to perform quantitative RT-PCR validation. All primers used in this study were listed in Table S1.

\subsection{Statistical Analysis}

Data were analyzed by one-way analysis of variance, followed by Dunnett's multiple comparison and Tukey's test using the SPSS v23.0 software. All results were expressed as the mean \pm standard error of the mean (SD). $p<0.05$ was considered statistically significant.

\section{Results}

\subsection{Phylogenetic Analysis of ILVC from Eukaryotes}

Phylogenetic analysis showed that ILVC protein from fungi, plants, and bacteria were clustered in respective clades. Analysis of the domain architecture showed that ILVC protein from plants had the shortest conserved domain (ILVC superfamily) and bacterial ILVC sequences were the longest in length among the eukaryotes (Figure 1A). 


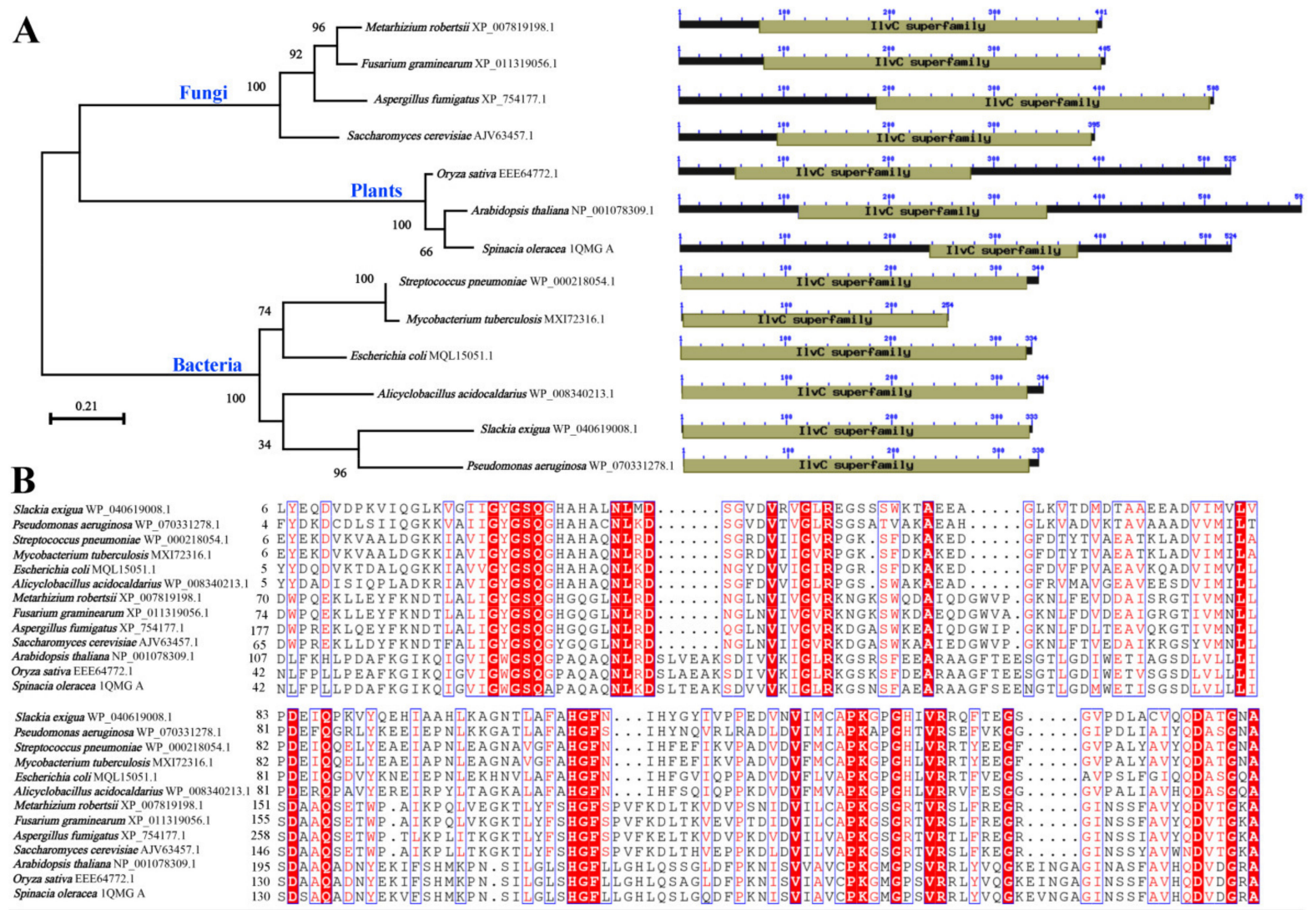

Figure 1. ILVCs in diverse organisms. (A) Phylogenetic analysis of ILVCs. (B) Conserved domain arrangements of the ILVCs.

Five amino acid residues contacting both NADP(H) and $\mathrm{Mg}^{2+}$ are also conserved in fungi, bacteria, and plants, indicating that all these ILVCs have either reductase or isomerase activity [11]. In $M$. robertsii, residues contacting both $\mathrm{NADP}(\mathrm{H})$ and $\mathrm{Mg}^{2+}$ are Arg-113, Ser-118, Asp-152, Asp-260, and Glu-264 (Figure 1B).

\subsection{Enzymatic Activity of ILVC from Different Fungi and MrILVC with Active-Site Mutant}

As shown in Figure 2A, ILVC from phytopathogenic fungi (A. fumigatus and F. graminearum) exhibited significantly higher activities than those from entomopathogenic fungi (B. bassiana and M. robertsii) but lower than that from S. cerevisiae.

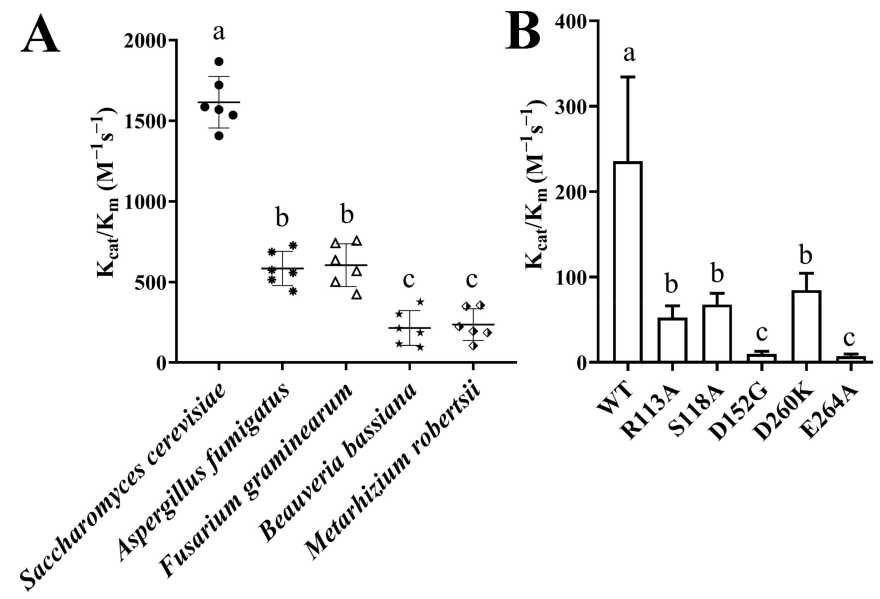

Figure 2. Kinetic parameters of ILVCs. (A) Kinetic parameters of different fungal ILVCs. (B) Kinetic parameters of MrILVC and its mutants. Different lowercase letters marked on the bars in each graph denote significant differences $(p<0.05)$. Error bars, standard deviation from six replicate assays. 
All the mutants (R113A, S118A, D152G, D260K, and E264A) exhibited significantly reduced activities compared to those of WT (Figure 2B), confirming that the roles of those residues on ILVC activities were conserved among different fungi [15].

\subsection{Deletion of MrILVC Increased Tolerance to AHAS Inhibitors}

All AHAS inhibitors showed significant inhibitory effects with $10 \sim 40 \%$ mycelial growth inhibition on the wild-type (Figure 3). Compared with the WT, $\triangle M$ MrilvC showed significant resistance to tribenuron-methyl, rimsulfuron, halosulfuron-methyl, chlorimuronethyl, imazethapyr, and imazapyr, but not metsulfuron-methyl, suggesting that those six AHAS inhibitors might inhibit the MrILVC activity.

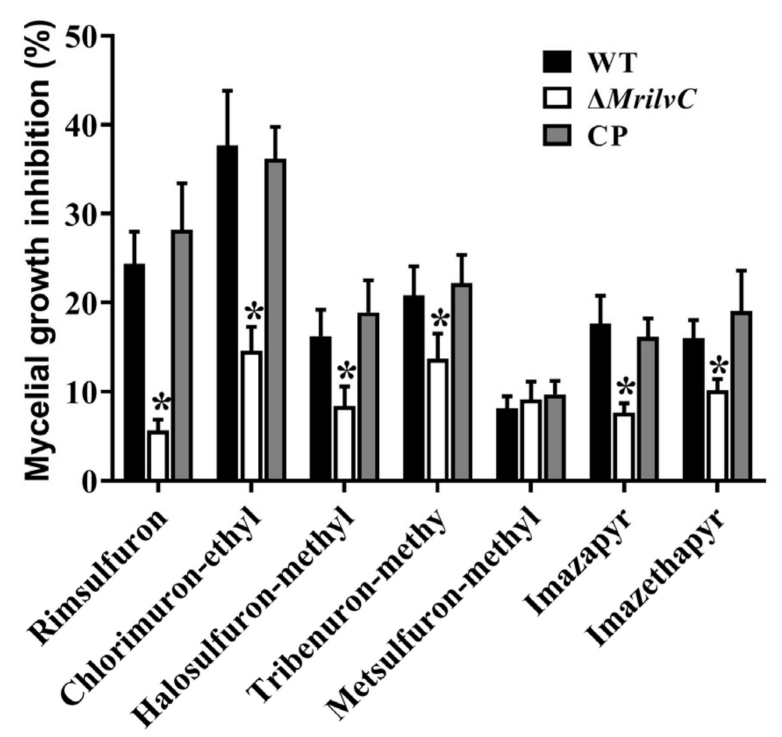

Figure 3. The MrILVC deletion mutant is resistant to AHAS inhibitors. Error bars represent the SEM of six replicate assays. * Significantly different expression with respect to the WT standard $(p<0.05)$.

\subsection{ILVC Contributes to Mycelial Growth and Conidial Germination}

MrilvC expression sharply increased expression at the stage of conidial germination and decreased rapidly from $60 \mathrm{~h}$ (Figure 4A). Additionally, MrilvC expression, increased rapidly after infection with conidia, was highly expressed from 96 h-I when M. robertsii has entered into the hemocoel.

Conidia of the $\triangle$ Mrilv $C$ mutant were unable to germinate on CZA medium supplied with all three BCAAs (Ile, Leu and Val), but were able to germinate on CZA medium supplemented with yeast extract (Figure 4B). The MrilvC-deficient strain was unable to grow on CZA plates, but could grow on CZA medium supplemented with BCAAs or yeast extract despite the colony being smaller than that of the WT (Figure 4C,D). In addition, in contrast to the WT and complemented transformant, the MrilvC-deficient strain showed a dramatic decrease in the conidial yields in CZA medium supplemented with BCAAs or yeast extract (Figure 4E).

\subsection{RNA-Seq Analysis of $\triangle$ MrilvC on Different Medium}

Totals of 10,934 (WT) and 10,869 (CZA) genes were detected in the WT and $\triangle$ MrilvC mutant grown on CZA plates; 11,011 (CZA + AA) and 11,012 (CZA + yeast) genes expressed in the $\triangle$ MrilvC mutant grow on CZA + AA and CZA + yeast plates (Figure 5A). Of these genes, 312 and 247 genes were found to be specifically expressed in WT and CZA, respectively, indicating the potential roles of MrILVC in their expression. In addition, 82, 87, and 111 genes were expressed specifically in CZA, CZA + AA and CZA + yeast samples, respectively. 

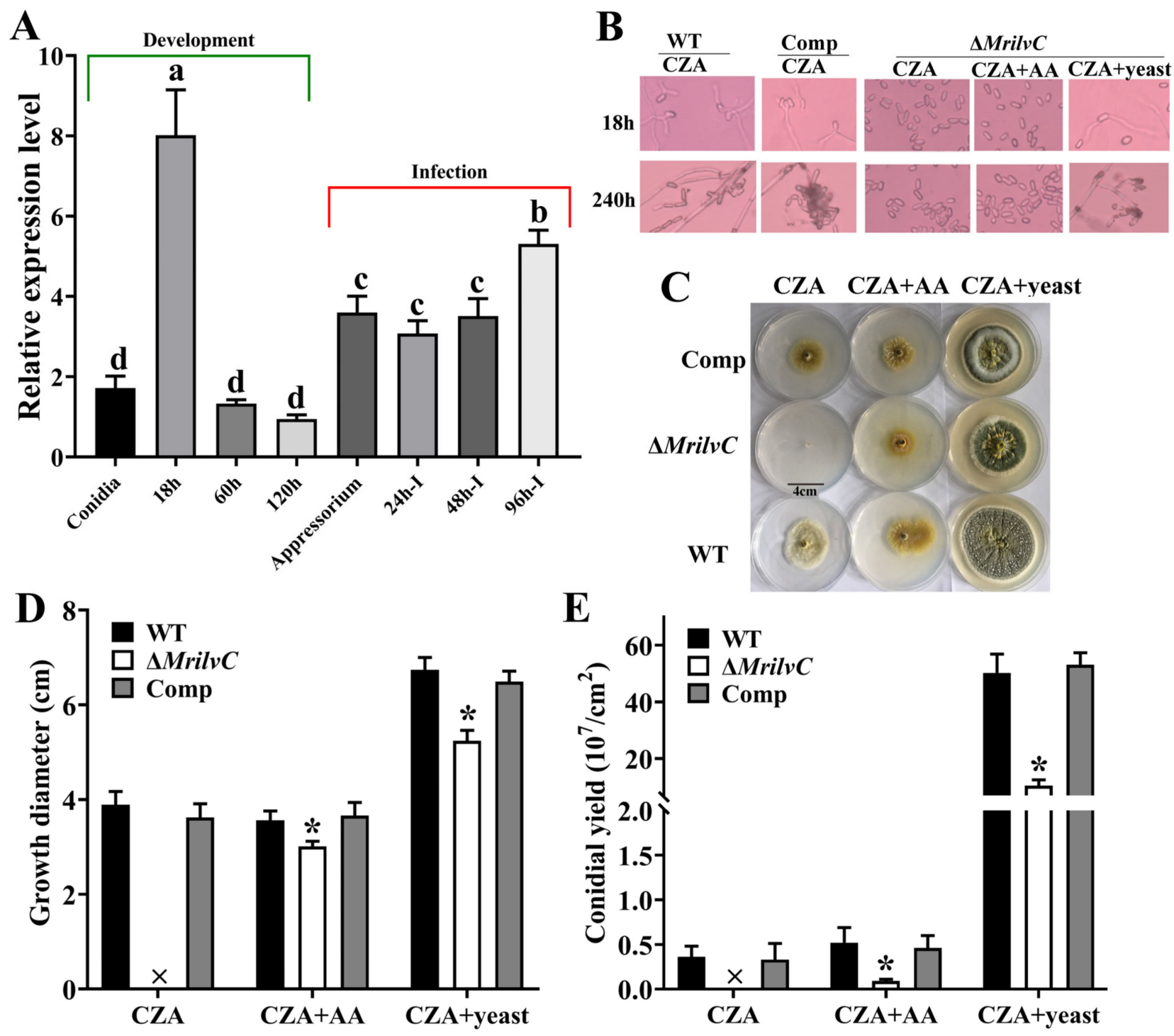

$\mathbf{E}$
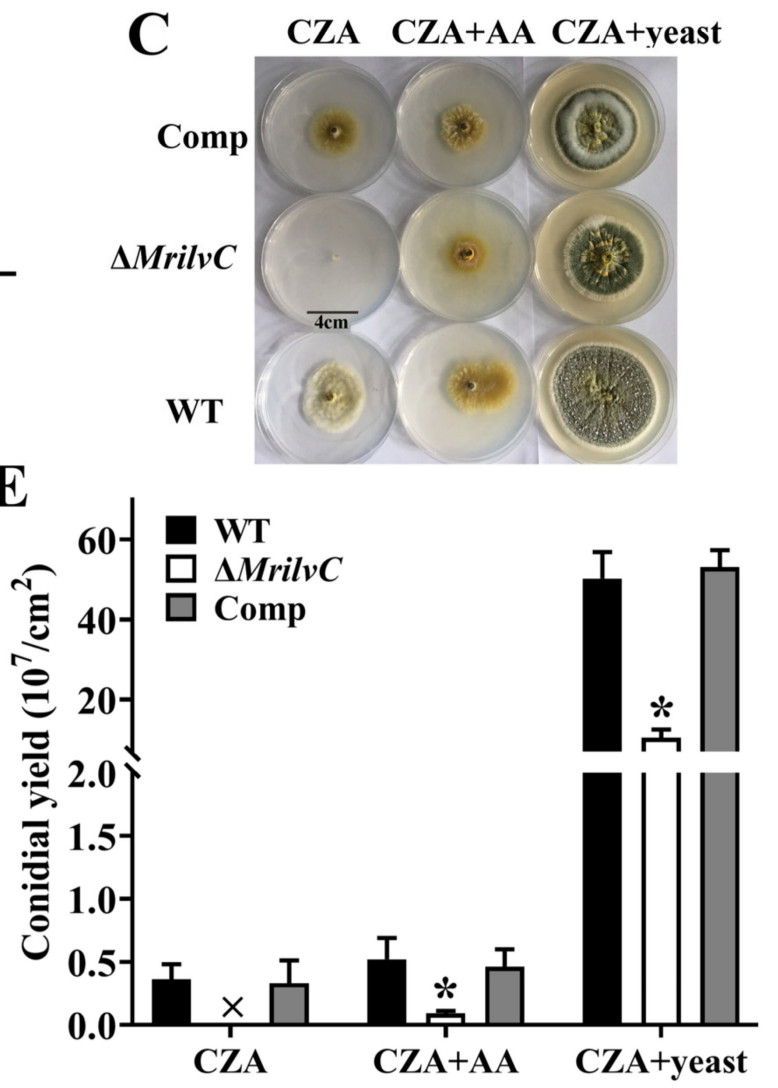

Figure 4. Deletion of MrILVC affected conidial germination, hyphal growth, and conidiation. (A) Transcriptional profiling of MrilvC at different developmental and infection stages. (B) Microscopy observation of WT and $\Delta$ MrilvC in different media. (C) Colony morphologies of WT and $\triangle M$ rilvC in different media. (D,E) Growth diameters and conidial yields of different strains cultured on CZA, CZA + AA, and CZA + yeast. Error bars represent the SEM of six replicate assays. * Significantly different expression $(p<0.05)$. Different lowercase letters marked on the bars in each graph denote significant differences $(p<0.05)$.

Compared with the WT, 1524 genes were significantly up-regulated while 2556 genes were significantly down-regulated after the deletion of MrilvC in M. robertsii grown on CZA plates (Figure $5 \mathrm{~B}$ ). It is worth mentioning that, compared with the WT, all genes encoding key enzymes of BCAA biosynthesis were significant up-regulated in the CZA, indicating that the disruption of BCAA metabolism contributes to the expression levels of those key enzymes involved in BCAA biosynthesis (Figure S1). Moreover, a total of 3165 and 4020 genes were differentially expressed between CZA + AA/CZA + yeast and CZA samples, respectively, and 2536 DEGs were found between CZA + AA and CZA + yeast samples. 
A
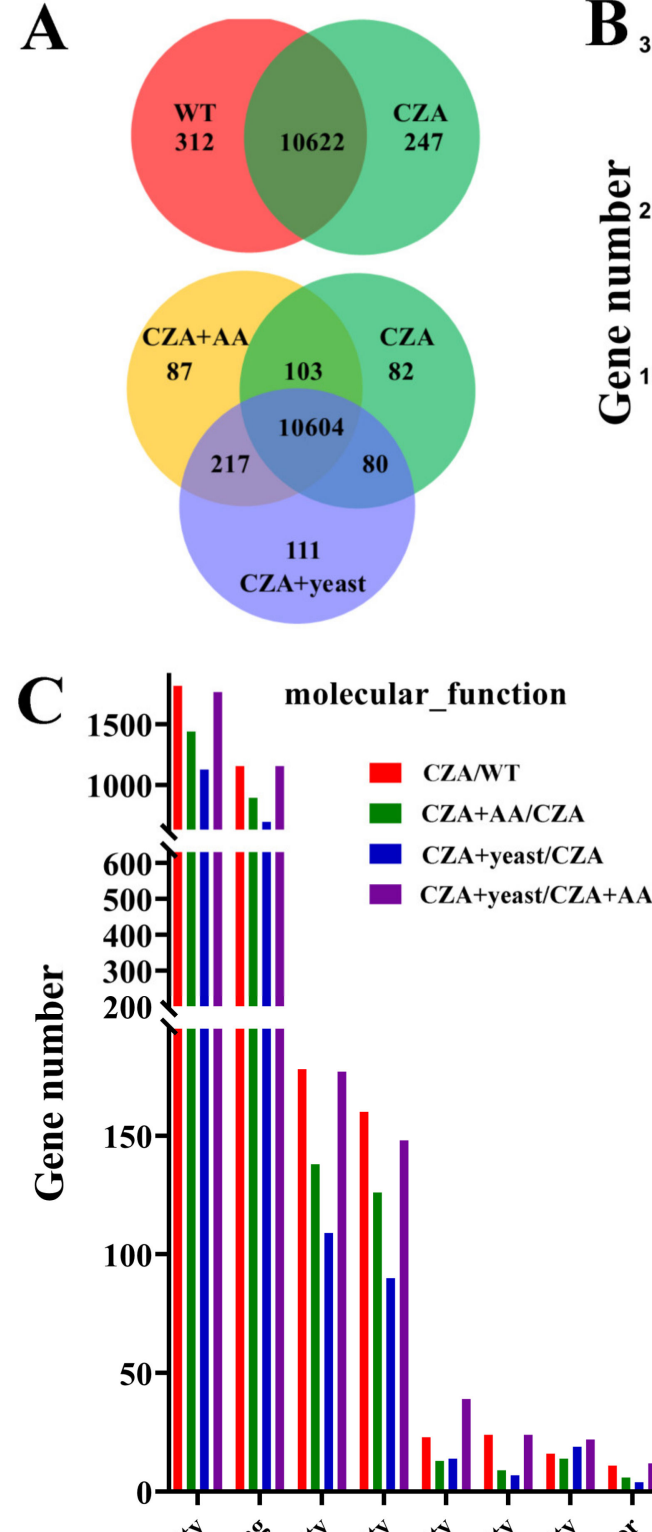

ícellular_componenti I<smiles>[10BH]</smiles>
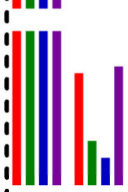

Up-regulated Down-regulated

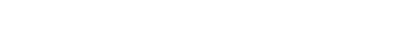

2531

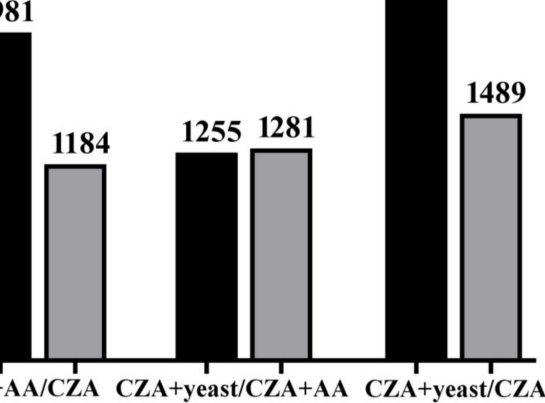

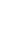

$$
\text { II I I I I }
$$

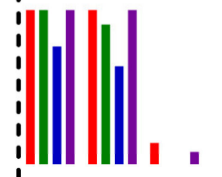

biological_process
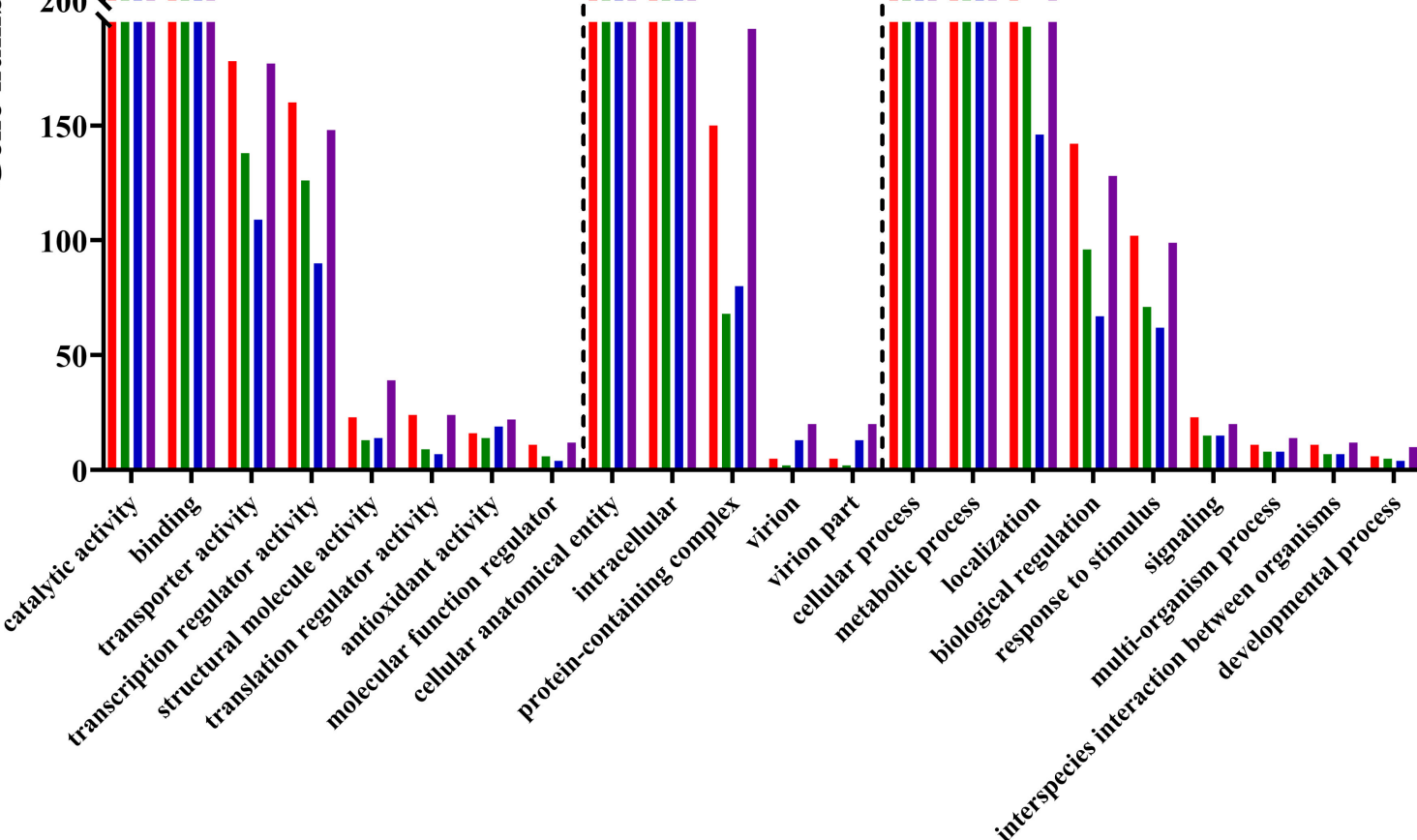

Figure 5. Gene expression in the wild-type strain cultured on czapek agar (WT) and $\triangle M$ rilvC cultured on czapek agar (CZA) supplemented with BCAAs (CZA + AA) or yeast extract (CZA + yeast). (A) Venn diagram showing the distribution of expressed genes. (B) DEGs from WT or CZA or CZA + AA or CZA + yeast samples. (C) GO enrichment analyses of the DEGs from different samples. WT: The WT strain cultured on CZA plates; CZA: $\triangle M$ rilvC mutant grown on CZA plates; $\mathrm{CZA}+\mathrm{AA}: \triangle M$ rilv C mutant grown on CZA + AA plates; CZA + yeast: $\triangle M r i l v C$ mutant grown on CZA + yeast plates.

Based on sequence homology, the sequences significantly enriched 22 functional groups (Figure 5C). Catalytic activity, binding, cellular anatomical entity, intracellular and cellular process, and metabolic process were the most commonly annotated terms in each of the three GO term categories. The DEGs of WT/CZA were enriched in 17 KEGG pathways $(p<0.05)$ (Table 1). Of these pathways, biosynthesis of antibiotics and biosynthesis of amino 
acids were the most highly enriched KEGG pathways, indicating that the severity affection of normal biosynthesis in M. robertsii is caused by the MrILVC deletion. The biosynthesis of antibiotics and biosynthesis of amino acids were also the most enriched pathways in $\mathrm{CZA}+\mathrm{AA} / \mathrm{CZA}$ and CZA + yeast/CZA, but not in CZA + yeast/CZA + AA. As mentioned above, plenty of DEGs, the expression levels of these genes were validated using quantitative RT-PCR, from WT/CZA (116), CZA + AA/CZA (95), and CZA + yeast/CZA (106) were enriched in the biosynthesis of amino acids. In addition to DEGs involved in BCAA biosynthesis, some DEGs participated in the biosynthesis of other amino acids, such as lysine, proline, and arginine (Table S2).

Table 1. KEGG analysis of differentially expressed genes.

\begin{tabular}{|c|c|c|c|c|c|}
\hline \multirow{2}{*}{$\begin{array}{l}\text { Pathway } \\
\text { ID }\end{array}$} & \multirow{2}{*}{ Pathway Name } & \multicolumn{4}{|c|}{ Gene Number } \\
\hline & & CZA/WT & $\mathbf{C Z A}+\mathbf{A A} / \mathbf{C Z A}$ & CZA + Yeast/CZA & $\mathrm{CZA}+$ Yeast $/ \mathrm{CZA}+\mathbf{A A}$ \\
\hline ko01230 & Biosynthesis of amino acids & 116 & 95 & 106 & - \\
\hline ko00260 & Glycine, serine, and threonine metabolism & 91 & 76 & 82 & 54 \\
\hline ko01130 & Biosynthesis of antibiotics & 267 & 221 & 259 & - \\
\hline ko00970 & Aminoacyl-tRNA biosynthesis & 51 & 39 & 47 & - \\
\hline ko01210 & 2-Oxocarboxylic acid metabolism & 45 & 35 & - & - \\
\hline ko00220 & Arginine biosynthesis & 26 & 22 & 22 & - \\
\hline ko00360 & Phenylalanine metabolism & 62 & 60 & 56 & 44 \\
\hline ko00910 & Nitrogen metabolism & 28 & 24 & 26 & - \\
\hline ko00410 & beta-Alanine metabolism & 54 & 44 & - & - \\
\hline ko00650 & Butanoate metabolism & 36 & 29 & - & - \\
\hline ko00630 & Glyoxylate and dicarboxylate metabolism & 43 & - & - & - \\
\hline ko00920 & Sulfur metabolism & 24 & - & 51 & - \\
\hline ko00310 & Lysine degradation & 47 & - & 40 & - \\
\hline ko00350 & Tyrosine metabolism & 63 & 55 & & - \\
\hline ko00740 & Riboflavin metabolism & 25 & - & 27 & - \\
\hline ko00750 & Vitamin B6 metabolism & 13 & - & - & - \\
\hline ko00290 & Valine, leucine, and isoleucine biosynthesis & 19 & 17 & - & - \\
\hline ko00380 & Tryptophan metabolism & - & 63 & 67 & 48 \\
\hline ko00400 & Phenylalanine, tyrosine, and tryptophan biosynthesis & - & 22 & 23 & - \\
\hline ko00250 & Alanine, aspartate, and glutamate metabolism & - & 41 & 46 & - \\
\hline ko00340 & Histidine metabolism & - & 21 & - & - \\
\hline ko00254 & Aflatoxin biosynthesis & - & 21 & - & - \\
\hline ko00603 & $\begin{array}{l}\text { Glycosphingolipid biosynthesis-globo and } \\
\text { isoglobo series }\end{array}$ & - & 6 & - & - \\
\hline ko02010 & $\mathrm{ABC}$ transporters & - & 28 & - & - \\
\hline ko00561 & Glycerolipid metabolism & - & 31 & 37 & - \\
\hline ko00052 & Galactose metabolism & - & 22 & - & - \\
\hline ko00330 & Arginine and proline metabolism & - & 41 & - & - \\
\hline ko00460 & Cyanoamino acid metabolism & - & 27 & 36 & - \\
\hline ko00300 & Lysine biosynthesis & - & 14 & - & - \\
\hline ko00520 & Amino sugar and nucleotide sugar metabolism & - & - & - & 109 \\
\hline ko00072 & Synthesis and degradation of ketone bodies & - & - & 11 & - \\
\hline ko00450 & Selenocompound metabolism & - & - & 12 & - \\
\hline ko01200 & Carbon metabolism & - & - & 96 & - \\
\hline ko00240 & Pyrimidine metabolism & - & - & 31 & - \\
\hline
\end{tabular}

WT: The WT strain cultured on CZA plates; CZA: $\triangle M$ rilvC mutant grown on CZA plates; CZA + AA: $\triangle M$ rilvC mutant grown on CZA + AA plates; $\mathrm{CZA}+$ yeast: $\triangle M$ rilv $C$ mutant grown on CZA + yeast plates.

Furthermore, DEGs from CZA + yeast/CZA + AA were mostly involved in amino sugar and nucleotide sugar metabolism (109), followed by those involved in glycine, serine, and threonine metabolism (58), tryptophan metabolism (48), and phenylalanine metabolism (44).

\subsection{MrILVC Affects Expression of Genes Involved in Mycelial Growth and Conidial Germination}

Venn analysis showed that a total of 459 DEGs were found between conidial germinated and inhibited samples (Figure 6A and Table S3). Among of them, more than 100 DEGs were significantly enriched in seven function classes with function classification, for which biosynthesis of antibiotics (40 DEGs) was the most highly enriched pathway. Based on the expression patterns, DEGs with higher expression levels in conidial germinated than 
inhibited samples were listed in Table 2. Among of them, catalase A and SAM-dependent methyltransferase genes played important roles in the fungal conidial germination [24,25].
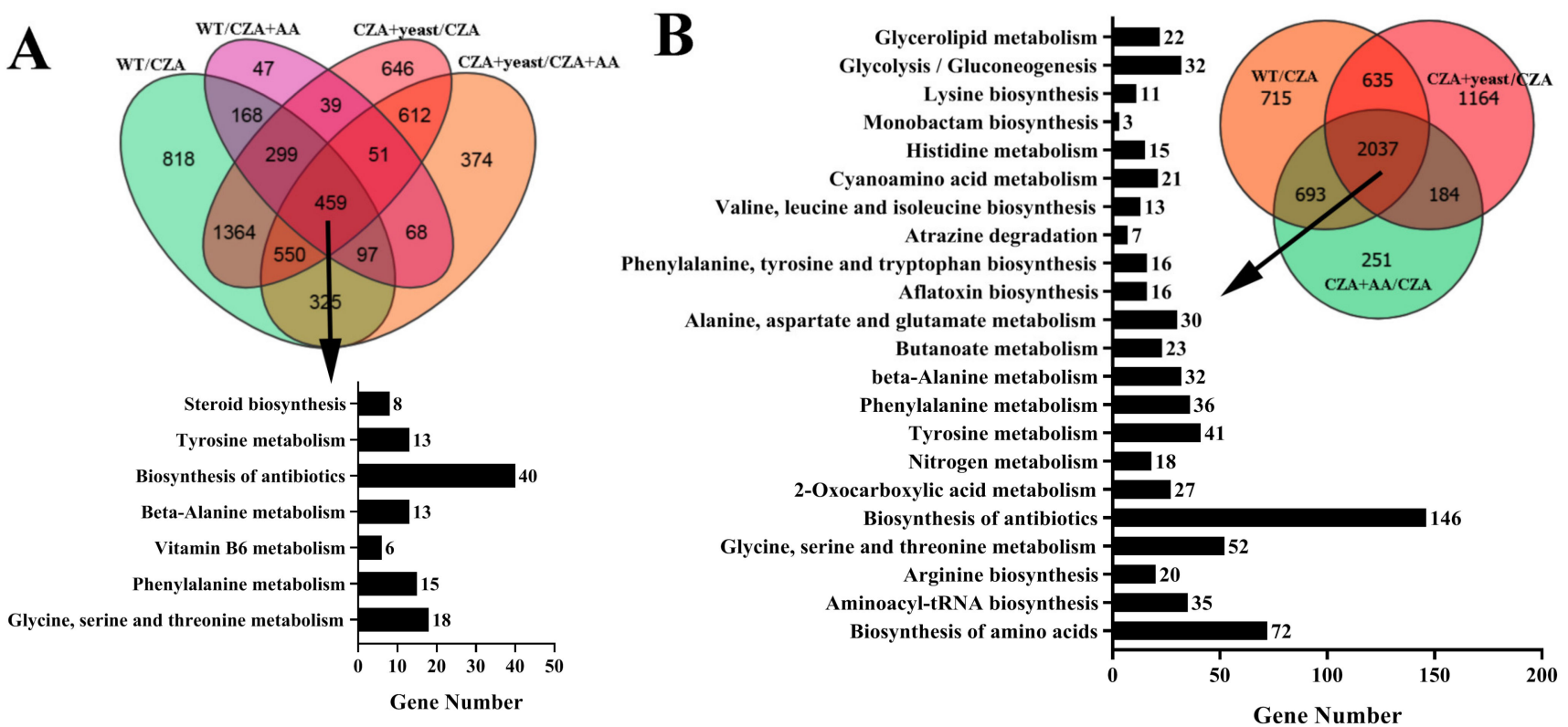

Figure 6. Venn diagrams of DEGs between conidial germinated and non-germinated samples (A) and mycelial growth and non-growth samples (B). DEGs in common were analyzed with KEGG enrichment. WT: The WT strain cultured on CZA plates; CZA: $\triangle M$ MrilvC mutant grown on CZA plates; CZA + AA: $\triangle M$ rilvC mutant grown on CZA + AA plates; CZA + yeast: $\triangle$ MrilvC mutant grown on CZA + yeast plates.

Table 2. Potential genes involved in conidial germination.

\begin{tabular}{|c|c|c|c|c|}
\hline \multirow{2}{*}{ Gene Name } & \multicolumn{4}{|c|}{ FPKM } \\
\hline & WT & CZA + Yeast & $\mathbf{C Z A}+\mathbf{A A}$ & CZA \\
\hline hypothetical protein & 214.29 & 706.56 & 103.95 & 20.36 \\
\hline catalase A & 99.73 & 59.86 & 19.71 & 4.41 \\
\hline DNA-binding WRKY domain-containing protein & 46.66 & 55.6 & 21.36 & 5.12 \\
\hline bacterial-type extracellular deoxyribonuclease & 55.33 & 21.65 & 8.84 & 3.24 \\
\hline hypothetical protein MAA_10685 & 22.67 & 44.16 & 8.14 & 0.1 \\
\hline macrophomate synthase & 32.71 & 31.71 & 6.15 & 0.08 \\
\hline Cytochrome P450 CYP684F1 & 30.19 & 11.43 & 1.82 & 0.16 \\
\hline NAD(P)-binding domain protein & 8.44 & 30.33 & 3.3 & 0.17 \\
\hline hypothetical protein & 6.03 & 31.47 & 2.19 & 0.33 \\
\hline lanosterol synthase & 8.76 & 30.28 & 2.79 & 2.82 \\
\hline HypA & 21.51 & 12.53 & 6.18 & 0.34 \\
\hline D-isomer specific 2-hydroxyacid dehydrogenase & 15.77 & 8.56 & 0.82 & 0.24 \\
\hline methyltransferase & 16.66 & 11.94 & 4.87 & 0.92 \\
\hline SAM-dependent methyltransferase & 16.22 & 10.06 & 2.6 & 1.32 \\
\hline NAD(P)-binding domain protein & 10.49 & 18.05 & 4.5 & 1.94 \\
\hline hypothetical protein & 5.59 & 18.58 & 2.04 & 0.37 \\
\hline bacterial-type extracellular deoxyribonuclease & 12.56 & 14.15 & 5.36 & 2.05 \\
\hline membrane copper amine oxidase & 9.93 & 9.4 & 3.94 & 1.79 \\
\hline NADP-dependent alcohol dehydrogenase C & 6.71 & 7.22 & 2.98 & 0 \\
\hline Cytochrome P450 CYP68N3 & 1.6 & 2.4 & 0.21 & 0 \\
\hline
\end{tabular}

WT: The WT strain cultured on CZA plates; CZA: $\triangle M$ rilvC mutant grown on CZA plates; CZA + AA: $\triangle M$ rilvC mutant grown on CZA + AA plates; CZA + yeast: $\triangle$ MrilvC mutant grown on CZA + yeast plates.

Venn analysis of DEGs showed 2037 DEGs co-existed in WT/CZA, CZA + AA/CZA, and CZA + yeast/CZA, and were significantly enriched in 22 function classes (Figure $6 \mathrm{~B}$ and Table S4). DEGs with higher expression levels in mycelial growth than inhibited samples 
were listed in Table 3. Some genes involved in mycelial growth, such as Alcohol dehydrogenase, catalase A and chitinase were lower expressed in CZA than other samples [26-28].

Table 3. Potential genes involved in mycelial growth.

\begin{tabular}{|c|c|c|c|c|}
\hline \multirow{2}{*}{ Gene Name } & \multicolumn{4}{|c|}{ FPKM } \\
\hline & WT & CZA + Yeast & $\mathbf{C Z A}+\mathbf{A A}$ & CZA \\
\hline sarcosine oxidase & 2856.28 & 3610.63 & 1698.84 & 765.77 \\
\hline $\begin{array}{l}\text { tryptophan synthase beta subunit-like PLP-dependent } \\
\text { enzyme }\end{array}$ & 220.33 & 216.52 & 115.84 & 5.72 \\
\hline hypothetical protein & 258 & 238.66 & 152.01 & 20.68 \\
\hline alcohol dehydrogenase superfamily, zinc-type & 265.12 & 239.15 & 182.46 & 42.76 \\
\hline catalase A & 99.73 & 59.86 & 19.71 & 4.41 \\
\hline macrophomate synthase & 32.71 & 31.71 & 6.15 & 0.08 \\
\hline hypothetical protein & 62.78 & 59.9 & 38.78 & 6.99 \\
\hline aldehyde dehydrogenase & 103.97 & 90.51 & 74.29 & 30.37 \\
\hline Chitinase & 72.67 & 31.01 & 15.75 & 0.3 \\
\hline bacterial-type extracellular deoxyribonuclease & 55.33 & 21.65 & 8.84 & 3.24 \\
\hline glutamate decarboxylase & 149.42 & 125.48 & 112.82 & 49.62 \\
\hline Amine oxidase & 38.32 & 37.46 & 25.08 & 3.05 \\
\hline hypothetical protein & 184.05 & 33.08 & 22.57 & 2.77 \\
\hline Cytochrome P450 CYP684F1 & 30.19 & 11.43 & 1.82 & 0.16 \\
\hline hypothetical protein & 31.27 & 19.17 & 10.99 & 0 \\
\hline hypothetical protein & 201.58 & 43.19 & 35.55 & 14 \\
\hline methyltransferase & 16.66 & 11.94 & 4.87 & 0.92 \\
\hline aldose 1-epimerase & 33.08 & 29.01 & 22.51 & 6.58 \\
\hline HypA & 21.51 & 12.53 & 6.18 & 0.34 \\
\hline membrane copper amine oxidase & 9.93 & 9.4 & 3.94 & 1.79 \\
\hline alpha/beta hydrolase fold-3 & 40.18 & 35.92 & 30.48 & 13.89 \\
\hline hypothetical protein & 18.13 & 6.68 & 3.1 & 0.36 \\
\hline cytochrome P450 CYP5321A1 & 54.37 & 34.38 & 32.75 & 1.61 \\
\hline peroxisomal copper amine oxidase & 2.92 & 2.07 & 1.96 & 0.9 \\
\hline
\end{tabular}

WT: The WT strain cultured on CZA plates; CZA: $\triangle M$ rilv C mutant grown on CZA plates; CZA + AA: $\triangle M$ rilvC mutant grown on CZA + AA plates; $C Z A+$ yeast: $\triangle M$ rilv C mutant grown on CZA + yeast plates.

In addition, 20 genes involved in conidial germination or mycelial growth were shown to be up-regulated in WT/CZA, CZA + AA/CZA, and CZA + yeast/CZA from RNA-Seq data. Quantitative RT-PCR analysis revealed 19, 18, and 18 genes were significantly upregulated in WT/CZA, CZA + AA/CZA, and CZA + yeast/CZA, respectively, supporting the validity of our transcriptomics results (Figure $\mathrm{S} 2$ ).

\section{Discussion}

ILVCs can be separated into two classes based on the length of their polypeptides: class I proteins with $\sim 340$ amino acid residues, and class II proteins with more than 520 residues [12,13]. In our study, ILVCs in fungi had various lengths from 400 to 500 residues, which were located between the plant and the bacteria in length. Domain architecture analysis showed that all ILVCs have the IlvC domain, and plant ILVCs have a C-terminal extension, play dual roles in forming the active site, but are missing in ILVCs of both bacteria and fungi, confirming that ILVCs from bacteria and fungi belong to the class I group and suggesting they may have similar function mechanisms which were different from plant ILVCs [13]. Moreover, multiple-sequence alignment of ILVCs showed that residues constituting $\mathrm{NADP}(\mathrm{H})$ and $\mathrm{Mg}^{2+}$ binding sites are well conserved, while the overall length of each KARI protein is different.

ILVCs from different organisms have evolved different mechanisms of induced fit to prepare the active site for catalysis [12]. Enzymatic activity assays showed that ILVC from phytopathogenic fungi exhibited significantly higher activities than those from entomopathogenic fungi but lower than that from S. cerevisiae, suggesting that there is a 
direct link between the ILVC catalysis activity and the characteristics of fungal life cycles. ILVC and its orthologous proteins have either reductase or isomerase activity, but only the reductase activity was explored in this study because of the difficulty and reaction complex of isomerase action [11,15]. Structure-based multiple sequence alignment of ILVCs from bacteria and plants revealed that five amino acid residues contacting both NADP $(\mathrm{H})$ and $\mathrm{Mg}^{2+}$ are conserved [15]. In our study, sequence alignment and enzymatic activities of MrILVC with different active-site mutants confirmed that those residues are also conserved in fungal ILVCs.

To date, BCAA enzyme inhibitors shown antimicrobial effects on some harmful pathogenic microorganisms, such as F. graminearum, P. aeruginosa, and C. albicans $[6,9,10]$. In this study, all tested AHAS inhibitors showed significant inhibitory effects with 10 40\% mycelial growth inhibition on $M$. robertsii, revealing that the use of entomopathogenic fungi to control pests should be avoided during the AHAS inhibitors' used area. Consistent with the observation in F. graminearum, deleting ilv $C$ showed significant resistance to tribenuronmethyl, rimsulfuron, halosulfuron-methyl, chlorimuronethyl, imazethapyr and imazapyr, suggesting that those six AHAS inhibitors might inhibit the fungal ILVC activity [6].

In this study, qRT-PCR showed MrilvC expression sharply increased expression at the stage of conidial germination and infection, which is consistent with the impairment of the full virulence on insects treated with direct injection or cuticle infection in our previous study [14]. BCAAs account for more than $20 \%$ of total protein amino acids and are an important constituent of the amino acid pool in organisms [29]. Transcriptomics analysis showed lots of genes involved in both metabolism and biosynthesis of amino acids were differentially expressed after the MrilvC deletion, which implied that the blocking of BCAAs impaired protein functioning in those pathways.

Catalase A, a monofunctional catalase in fungi, played crucial roles in various aspects of cell physiology and cellular differentiation, including conidial germination and mycelial growth $[25,30]$. In $M$. robertsii, the expression of catalase $A$ was down-regulated after the deletion of MrilvC, while it returned to an expression level similar to the WT in $\triangle$ MrilvC feeding with yeast extract. In addition, some genes involved in conidial germination or mycelial growth, such as Alcohol dehydrogenase, SAM-dependent methyltransferase, and chitinase, showed similar expression patterns with catalase A. From these results, MrilvC played critical roles in fungal development by regulating those gene expressions, and characteristics of $\triangle M$ rilv $C$ could be partially restored to the WT with gene regulation by feeding with BCAAs or yeast extract. It is worth mentioning that deleting MrilvC led to the noticeable increase in the expression of genes involved in BCAA biosynthesis, and we speculated that BCAA levels could affect its biosynthesis and the disruption of BCAA biosynthesis by deleting $M r i l v C$, resulting in those genes being significant up-regulated; more experiments are needed to explore those patterns.

In conclusion, our data indicate that MrILVC plays critical roles in controlling conidial germination and mycelial growth by the regulation of metabolism and biosynthesis of amino acids. ILVCs from different fungi revealed distinct reductase activity. Active sites of MrILVC are conserved with plant and bacterial ILVCs; one mutation among those exhibited significantly reduced activities. Data from this study advance our understanding of the function of ILVCs in entomopathogenic fungi, which would contribute to future BCAA metabolism investigations.

Supplementary Materials: The following are available online at https:/ / www.mdpi.com/article/10 .3390/jof7090737/s1, Figure S1: Expression levels of genes involved in BCAA pathways from different samples, Figure S2: Quantitative RT-PCR validation of differential expressed genes identified in RNA-Seq analysis. WT: The WT strain cultured on CZA plates; CZA: $\triangle$ MrilvC mutant grow on CZA plates; CZA + AA: $\triangle$ MrilvC mutant grow on CZA + AA plates; CZA + yeast: $\triangle$ MrilvC mutant grow on CZA + yeast plates, Table S1: Primers used in this study, Table S2: Expression of genes involved in biosynthesis of amino acids, Table S3: Differential expressed genes between conidial germination and inhibited samples, Table S4: Differential expressed genes between mycelial growth and inhibited samples. 
Author Contributions: Conceptualization, B.H.; methodology, Y.W., X.Y. and S.L.; software, Y.W. and S.L.; validation, D.Y. and X.X.; formal analysis, B.H.; investigation, Y.W., X.Y. and S.L.; data curation, D.Y., X.X. and B.H.; writing-review and editing, Y.W. and B.H.; supervision, B.H.; project administration, B.H.; funding acquisition, B.H. All authors have read and agreed to the published version of the manuscript.

Funding: This work was supported by the Scientific Research Foundation of Anhui Agricultural University (Grant No. yj2019-09), Natural Science Foundation of the Anhui Higher Education Institutions (KJ2020A0129) and China Postdoctoral Science Foundation (Grant No. 2020M671838).

Institutional Review Board Statement: Not applicable.

Informed Consent Statement: Not applicable.

Data Availability Statement: Not applicable.

Conflicts of Interest: The authors declare no conflict of interest. The funders had no role in the design of the study; in the collection, analyses, or interpretation of data; in the writing of the manuscript; or in the decision to publish the results.

\section{References}

1. Neinast, M.; Murashige, D.; Arany, Z. Branched Chain Amino Acids. Annu. Rev. Physiol. 2019, 81, 139-164. [CrossRef]

2. Steyer, J.T.; Downes, D.J.; Hunter, C.C.; Migeon, P.A.; Todd, R.B. Duplication and functional divergence of branched-chain amino acid biosynthesis genes in Aspergillus nidulans. mBio 2021, 12, e00768-21. [CrossRef]

3. Mccourt, J.A.; Duggleby, R.G. Acetohydroxyacid synthase and its role in the biosynthetic pathway for branched-chain amino acids. Amino Acids 2006, 31, 173-210. [CrossRef] [PubMed]

4. Binder, S. Branched-chain amino acid metabolism in Arabidopsis thaliana. Arabidopsis Book Am. Soc. Plant Biol. $2010,8, \mathrm{e} 0137$.

5. Tan, S.; Evans, R.; Singh, B. Herbicidal inhibitors of amino acid biosynthesis and herbicide-tolerant crops. Amino Acids 2006, 30, 195-204. [CrossRef]

6. Liu, X.; Wang, J.; Xu, J.; Shi, J. FgIlv5 is required for branched-chain amino acid biosynthesis and full virulence in Fusarium graminearum. Microbiology 2014, 160, 692-702. [CrossRef]

7. Duggleby, R.G.; McCourt, J.A.; Guddat, L.W. Structure and mechanism of inhibition of plant acetohydroxyacid synthase. Plant Physiol. Biochem. 2008, 46, 309-324. [CrossRef] [PubMed]

8. Shimizu, M.; Fujii, T.; Masuo, S.; Takaya, N. Mechanism of de novo branched-chain amino acid synthesis as an alternative electron sink in hypoxic Aspergillus nidulans cells. Appl. Environ. Microb. 2010, 76, 1507-1515. [CrossRef]

9. Lee, Y.T.; Cui, C.J.; Chow, E.; Pue, N.; Lonhienne, T.; Wang, J.G.; Fraser, J.A.; Guddat, L.W. Sulfonylureas have antifungal activity and are potent inhibitors of Candida albicans acetohydroxyacid synthase. J. Med. Chem. 2013, 56, 210-219. [CrossRef] [PubMed]

10. Garcia, M.; Chua, S.; Low, Y.S.; Lee, Y.T.; Guddat, L.W. Commercial AHAS-inhibiting herbicides are promising drug leads for the treatment of human fungal pathogenic infections. Proc. Natl. Acad. Sci. USA 2018, 115, E9649-E9658. [CrossRef]

11. Tyagi, R.; Lee, Y.-T.; Guddat, L.W.; Duggleby, R.G. Probing the mechanism of the bifunctional enzyme ketol-acid reductoisomerase by site-directed mutagenesis of the active site. FEBS J. 2005, 272, 593-602. [CrossRef]

12. Wong, S.H.; Lonhienne, T.; Winzor, D.J.; Schenk, G.; Guddat, L.W. Bacterial and plant ketol-acid reductoisomerases have different mechanisms of induced fit during the catalytic cycle. J. Mol. Biol. 2012, 424, 168-179. [CrossRef]

13. Ahn, H.J.; Su, J.E.; Yoon, H.J.; Lee, B.I.; Cho, H.; Suh, S.W. Crystal structure of class i acetohydroxy acid isomeroreductase from Pseudomonas aeruginosa. J. Mol. Biol. 2003, 328, 505-515. [CrossRef]

14. Luo, F.; Zhou, H.; Zhou, X.; Xie, X.; Li, Y.; Hu, F.; Huang, B. The intermediates in branched-chain amino acid biosynthesis are indispensable for conidial germination of the insect-pathogenic fungus Metarhizium robertsii. Appl. Environ. Microbiol. 2020, 86, e01682-20. [CrossRef]

15. Kim, G.; Shin, D.; Lee, S.; Yun, J.; Lee, S. Crystal structure of ilvc, a ketol-acid reductoisomerase, from Streptococcus Pneumoniae. Crystals 2019, 9, 551. [CrossRef]

16. Wang, Z.; Meng, H.; Zhuang, Z.; Chen, M.; Bo, H. Molecular cloning of a novel subtilisin-like protease (Pr1A) gene from the biocontrol fungus Isaria farinosa. Appl. Entomol. Zool. 2013, 48, 477-487. [CrossRef]

17. Brinkmann-Chen, S.; Flock, T.; Cahn, J.; Snow, C.D.; Brustad, E.M.; Mcintosh, J.A.; Meinhold, P.; Zhang, L.; Arnold, F.H. General approach to reversing ketol-acid reductoisomerase cofactor dependence from NADPH to NADH. Proc. Natl. Acad. Sci. USA 2013, 110, 10946-10951. [CrossRef]

18. Li, K.-H.; Yu, Y.-H.; Dong, H.-J.; Zhang, W.-B.; Ma, J.-C.; Wang, H.-H. Biological functions of ilvc in branched-chain fatty acid synthesis and diffusible signal factor family production in Xanthomonas campestris. Front. Microbiol. 2017, 8, 2486. [CrossRef]

19. Wang, Z.; Feng, J.; Jiang, Y.; Xu, X.; Xu, L.; Zhou, Q.; Huang, B. MrPEX33 is involved in infection-related morphogenesis and pathogenicity of Metarhizium robertsii. Appl. Microbiol. Biotech. 2021, 3, 1079-1090. [CrossRef]

20. Wang, Y.; Xie, X.; Qin, L.; Yu, D.; Wang, Z.; Huang, B. Integration of dsRNA against host immune response genes augments the virulence of transgenic Metarhizium robertsii strains in insect pest species. Microb. Biotech. 2021, 14, 1433-1444. [CrossRef] 
21. Wang, Y.; Zhou, Q.; Zhang, H.; Qin, L.; Huang, B. Immunotranscriptome analysis of Plutella xylostella reveals differences in innate immune responses to low- and high-virulence Beauveria bassiana strain challenges. Pest Manag. Sci. 2021, 77, 1070-1080. [CrossRef]

22. Mortazavi, A.; Williams, B.A.; McCue, K.; Schaeffer, L.; Wold, B. Mapping and quantifying mammalian transcriptomes by RNA-Seq. Nat. Methods 2008, 5, 621-628. [CrossRef]

23. Audic, S.p.; Claverie, J.M. The significance of digital gene expression profiles. Genome Res. 1997, 7, 986-995. [CrossRef]

24. Bello, M.H.; Barrera-Perez, V.; Morin, D.; Epstein, L. The Neurospora crassa mutant NcDEgt-1 identifies an ergothioneine biosynthetic gene and demonstrates that ergothioneine enhances conidial survival and protects against peroxide toxicity during conidial germination. Fungal Genet. Biotech. 2012, 49, 160-172. [CrossRef]

25. Baltussen, T.J.H.; Zoll, J.; Verweij, P.E.; Melchers, W.J.G. Molecular mechanisms of conidial germination in Aspergillus. Microbiol. Mol. Biol. Rev. 2020, 84, e00049-19. [CrossRef]

26. Jin, D.; Sun, B.; Zhao, W.; Ma, J.; Zhou, Q.; Han, X.; Mei, Y.; Fan, Y.; Pei, Y. Thiamine-biosynthesis genes Bbpyr and Bbthi are required for conidial production and cell wall integrity of the entomopathogenic fungus Beauveria bassiana. J. Invertebr. Pathol. 2021, 184, 107639. [CrossRef]

27. Zhang, E.; Cao, Y.; Xia, Y. Ethanol dehydrogenase I contributes to growth and sporulation under low oxygen condition via detoxification of acetaldehyde in Metarhizium acridum. Front. Microbiol. 2018, 9, 1932. [CrossRef]

28. Hernandez, C.E.M.; Guerrero, I.E.P.; Hernandez, G.A.G.; Solis, E.S.; Guzman, J.C.T. Catalase overexpression reduces the germination time and increases the pathogenicity of the fungus Metarhizium anisopliae. Appl. Microbiol. Biotech. 2010, 87, 1033-1044. [CrossRef]

29. Garault, P.; Letort, C.; Juillard, V.; Monnet, V. Branched-chain amino acid biosynthesis is essential for optimal growth of Streptococcus thermophilus in milk. Appl. Environ. Microbiol. 2000, 66, 5128-5133. [CrossRef]

30. Paris, S.; Wysong, D.; Debeaupuis, J.-P.; Shibuya, K.; Philippe, B.; Diamond, R.D.; Latgé, J.-P. Catalases of Aspergillus fumigatus. Infect. Immun. 2003, 71, 3551-3562. [CrossRef] 